\title{
REVISIÓN DE TEMA
}

Recibido para publicación: septiembre 30 de 2011.

Aceptado en forma revisada: octubre 30 de 2011.

\section{Contaminación de desinfectantes usados en el área quirúrgica ¿potencial bacteriano intrínseco, resistencia adquirida o factores extrínsecos relacionados?}

\author{
contamination of disinfectants used in the surgical area: intrinsic bacterial \\ potential, acquired resistance or related extrinsic factors?
}

\author{
Lorduy Gómez, Jaime; ; Carrillo González, Stephanye²
}

\begin{abstract}
RESUMEN
Los protocolos de descontaminación de instrumental quirúrgico o equipos de diagnóstico por técnicas de inmersión en desinfectantes fueron creados con el objetivo de disminuir la adquisición y diseminación de infecciones del sitio quirúrgico. La problemática relacionada con la resistencia que ejercen las bacterias a los antibióticos se ha extendido al uso de desinfectantes en cirugía, los microorganismos también han sido capaces de evadir su acción, tanto así que en la actualidad muchas cepas hospitalarias, presentan una resistencia cruzada a la acción de desinfectantes y antibióticos. De manera que es importante considerar los factores intrínsecos o adquiridos que están relacionados con la contaminación de estas sustancias biocidas, sin dejar de lado algunos factores externos relacionados.
\end{abstract}

Palabras Claves: Desinfección, endoscopios, instrumental quirúrgico, quirófanos.

\begin{abstract}
The protocols for decontamination of surgical instruments and diagnostic equipment in disinfectant immersion techniques were created with the aim of reducing the acquisition and dissemination of surgical site infections. The problems associated with resistance exerted by the bacteria to antibiotics has spread the use of disinfectants in surgery, microorganisms have also been able to evade their action, so much so that today many hospital strains, exhibit

\footnotetext{
${ }^{1}$ Bacteriólogo. Esp. en Microbiología Clínica. Programa de Instrumentación Quirúrgica, Corporación Universitaria Rafael Núñez.

${ }^{2}$ Instrumentadora Quirúrgica. Esp. en Bioética. Programa de Instrumentación Quirúrgica, Corporación Universitaria Rafael Núñez.

Correspondencia: jaime.lorduy@curnvirtual.edu,co
} 
https://doi.org/10.22519/21455333.302

crossresistance to action of disinfectants and antibiotics. So it is important to consider the intrinsic or acquired that are related to the contamination of biocides, without ignoring external factors involved.

Keywords: Disinfection, endoscopes, surgical instruments, operating room.

\section{INTRODUCCIÓN}

En los Estados Unidos, aproximadamente 46.5 millones de procedimientos quirúrgicos invasivos, e incluso los procedimientos médicos, incluyendo aproximadamente 5 millones de endoscopia gastrointestinal, se realizan cada año. Cada procedimiento consiste en poner en contacto un dispositivo médico o instrumento quirúrgico con tejido estéril de un paciente o de las membranas mucosas. Un riesgo importante de todos estos procedimientos es la introducción de patógenos que pueden conducir a la infección [1]. Se requiere entonces de una mejoría en los protocolos de descontaminación por técnicas de inmersión, que asegure que el procedimiento quirúrgico no representa un riesgo para la salud del paciente [2-3] todo esto considerando que el uso continuo de esterilizadores por calor es inadecuado para la eliminación de estos microorganismos; ya que causa daños estructurales en los lentes de los equipos de laparoscopia. La problemática relacionada con la resistencia que ejercen las bacterias a los antibióticos se ha extendido al uso de desinfectantes en cirugía, los microorganismos también han sido capaces de evadir su acción, tanto así que en la actualidad muchas cepas hospitalarias, presentan una resistencia cruzada a la acción de desinfectantes y antibióticos [4]. De manera que es importante considerar los factores intrínsecos o adquiridos que están relacionados con la contaminación de estas sustancias biocidas, sin dejar de lado que algunos factores externos como la preparación, uso y utilización de estas sustancias, son las que quizás estén más relacionados con la contaminación bacteriana [5].

\section{NECESIDAD EN EL USO DE MÉTODOS DE INMERSIÓN EN LA DESINFECCIÓN}

El método más común y accesible, utilizado en la mayoría de los hospitales, para la esterilización, es el vapor de agua a presión; aunque el uso de materiales poco resistentes al calor es cada vez más frecuente en la práctica médica. Esto ha obligado a los hospitales a adoptar otras alternativas de esterilización en frío, tales como el Oxido de Etileno, Irradiación Gamma y Gas Plasma de Peróxido de Hidrógeno, que son procesos difíciles y costosos, por lo tanto poco accesibles a la mayoría de los hospitales. Además, estos procesos requieren mucho tiempo, personal altamente capacitado y normas muy estrictas, tanto en la instalación del equipo como en el manejo de los productos de desecho de cada proceso [6]. Las mejoras tecnológicas en los sistemas ópticos operatorios y de diagnóstico, así como en el instrumental quirúrgico, requieren la utilización de materiales como plásticos y fibra óptica en su fabricación. Muchos de estos materiales no resisten la esterilización por vapor a presión, por lo que se requiere de métodos alternativos que sean rápidos, de bajo costo y altamente efectivos, características que se han logrado con los métodos de esterilización por inmersión en sustancias químicas [7]. La fibra óptica por ejemplo, es un componente fundamental de 
los equipos de endoscopia, con su uso se ha mejorado la visibilidad diagnóstica y operatoria. Sin embargo, se requiere de una mejoría en los protocolos de descontaminación y esterilización, ya que el calor a presión opacifica y disminuye la transmisión de la luz en dichos equipos.

Un gran problema con los endoscopios flexibles y con casi todos los rígidos es que, por sus costos actuales de fabricación, es imposible hacerlos desechables. Para el caso de broncoscopios por ejemplo, es importante el uso de técnicas de desinfección por inmersión que sean seguras, ya que se han reportado brotes de infección por gérmenes en su mayoría intrahospitalarios como Pseudomona aeruginosa que se encontraron en las soluciones usadas para su desinfección [8]. En el año 1961 Earle H. Spaulding ideó una clasificación para la desinfección y la esterilización de los elementos y equipos usados con el paciente. Esto resultó tan lógico, que se utilizó en todo el mundo como modelo para entender ambos procesos. La teoría de Spaulding se fundamenta en dividir los elementos de cuidado del paciente en tres categorías, basadas en el riesgo de infección que representan como críticos, semicríticos y no críticos. Se entiende por material crítico todo aquel que entre en contacto con tejidos estériles o con el sistema vascular, como por ejemplo endoscopios, instrumental quirúrgico, artroscopio, laparoscopio, toracoscopio, mediastinoscopio entre otros. Los semicríticos, son los que están en contacto con membranas, mucosas o piel no intacta, como por ejemplo aparatos de endoscopia rígidos que penetran en cavidades no estériles tales como: broncoscopio, rectoscopio, laringoscopio endoscopios flexibles de fibra óptica como broncoscopio, gastroscopio, colonoscopio, palas de laringoscopio; en los que se usa desinfección de alto nivel y el tiempo de exposición puede variar entre 20 y 30 minutos. Los no críticos, considerados aquellos que están en contacto con piel intacta, no con membranas mucosas y se usa desinfección de medio y bajo nivel [9].

En la mayoría de los hospitales se cuenta con un número limitado de equipos, lo que no permite una esterilización utilizando este tipo de procedimientos. Por esta razón el proceso de esterilización y descontaminación de equipos, accesorios de endoscopia en cirugía mínimamente invasiva y actualmente en el apoyo de microcirugía oftalmológica, se utiliza como desinfección la técnica de inmersión, la mayor parte de las veces dentro de la sala de operaciones o en áreas cercanas a esta [10-11].

\section{CONTAMINACIÓN BACTERIANA DE DESINFECTANTES DEL ÁREA QUIRÚRGICA}

La contaminación bacteriana de los desinfectantes los ha convertido en los vehículos ocasionales de infecciones hospitalarias durante más de 50 años. Existen algunos informes de epidemias por infecciones bacterianas relacionados con desinfectantes contaminados, estos principalmente utilizados para descontaminar instrumental quirúrgico y aparatos utilizados para el diagnóstico y tratamiento [12-14]. También se ha informado que desinfectantes contaminados presentan disminución de eficacia y efectividad para seguir eliminando agentes patógenos [15]. Un ejemplo de este tipo de estudios fue el realizado en el Instituto Nacional de Perinatología de México, en donde se recolectaron muestras microbiológicas del lavado de trocares reutilizados en laparoscopia, se detectaron cuatro aislamientos microbiológicos lo que corresponde a 
una tasa de infección de 3.8 por cada 100 cirugías laparoscópicas contaminadas. En el se aislaron microorganismos como Pseudomona aeruginosa, Enterococos sp, Enterococos faecalis y Bacillus $s p$ [16], los cuales se reconocen como agentes implicados en infecciones asociadas al cuidado de la salud. Sin embargo hoy, gérmenes que habitualmente hacen parte de la microbiota normal, como Staphilococcos coagulasa negativos, han demostrado resistencia a la acción de antisépticos y desinfectantes [17].

La contaminación de los desinfectantes es más frecuente de lo que habitualmente se cree y como resultado de esto se presenta infección en los pacientes. Se han informado casos en los que incluso las soluciones madre diluidas de desinfectantes presentaron contaminación antes de tener contacto con equipos de cirugía e instrumental quirúrgico [18]. Con la aparición del Virus de Inmunodeficiencia Humana (VIH) cambiaron incluso muchos procedimientos médicos que antes no se consideraban de alto riesgo. Una encuesta de las unidades de endoscopia en Universidades de Estados Unidos indicó que en la mayoría de estos centros, el personal que asiste a consulta, estaba realmente preocupado por el riesgo de contraer el SIDA [19].

Aunque el riesgo real sea difícil de determinar o aparentemente sea muy bajo, la importancia de la desinfección y esterilización de estos instrumentos médicos juega un papel importante para devolver la confianza en estos pacientes y garantizar la seguridad de los procedimientos. En Colombia, las infecciones causadas por una mala desinfección de dispositivos y objetos usados en cirugías aumentan de manera considerable los costos en la atención. Estudios en hospitales de Cundinamarca demuestran la presencia de microorganismos en el equipo de anestesia siendo los más frecuentes la Burkholderia cepacia con un 18,18\% [20]. Es importante aclarar que este tipo de gérmenes por sus escasos requerimientos nutricionales y su multirresistencia antibiótica podrían estar relacionados con infecciones asociadas al cuidado de la salud, sobre todo si contaminan desinfectantes de uso quirúrgico.

\section{FACTORES EXTRÍNSECOS RELACIONADOS CON LA CONTAMINACIÓN BACTERIANA DE DESINFECTANTES DEL ÁREA QUIRÚRGICA}

Dentro de las características asociadas a factores extrínsecos relacionadas con la contaminación bacteriana de desinfectantes se encuentran principalmente la capacitación del personal; respecto al uso, manejo de los desinfectantes y el empleo de elementos de protección personal, de la misma forma la verificación de las condiciones de almacenamiento; que conserven las condiciones del fabricante y el manejo del producto desinfectante, respecto a; modo de preparación, tiempo de preparación, tiempo de contacto, poco cuidado con recipientes contaminados, concentración y actividad del desinfectante, entre otros [21-22]. Muchos estudios evidencian la contaminación bacteriana de desinfectantes. Algunos de ellos tomaron muestras de soluciones de uso hospitalario y otros intencionalmente las contaminaron con bacterias para evaluar su efectividad.

Un estudio reciente, en el que se utilizaron soluciones biocidas de uso hospitalario realizado en Tailandia, se relacionó la contaminación bacteriana de desinfectantes con los factores externos relacionados. Esta evaluación se llevó a cabo mediante el cultivo 
de soluciones en uso y dentro de los factores extrínsecos relacionados se encuentran: la preparación de soluciones por personal no calificado, uso de contenedores inadecuados y el uso prolongado de desinfectantes.

La contaminación con bacterias se encontró en el 1,8\% de 16.142 muestras. La contaminación bacteriana de desinfectantes fue mayor en hospitales provinciales y no se ha encontrado en los hospitales universitarios. Los índices de contaminación se relacionaron más con la duración del uso y la mayoría de las bacterias aisladas fueron las que se encuentran en el medio ambiente [23]. Se ha documentado que las soluciones madre de desinfectantes vendidas comercialmente y preparadas en el departamento de farmacia, para luego ser distribuidas a las distintas unidades de hospitalización, han estado expuestas a contaminación [24-25]. Sin embargo en otro estudio realizado en la isla caribeña de Trinidad, las soluciones madre de los desinfectantes probados en todas las farmacias estaban libres de bacterias aerobias. Esto podría explicarse en parte por la alta concentración del principio activo en los desinfectantes antes de la dilución, así como el hecho de que aún no habían sido expuestos a la contaminación ambiental potencial. En este estudio se encontró que las prácticas sanitarias durante la preparación, el uso de contenedores no estériles y la distribución de desinfectantes antisépticos contribuyeron significativamente a la contaminación de desinfectantes en el medio hospitalario [18].

El uso de envases contaminados es un factor extrínseco importante; los resultados de algunas investigaciones con antisépticos permiten recomendar la limpieza como procedimiento mínimo en el procesamiento de esos recipientes que garantice la seguridad de su utilización repetida para distribución de estos y otros biocidas [26]. La cantidad de tiempo que las muestras diluidas se utilizan en las salas de cirugías puede ser el factor extrínseco mas asociado a la contaminación y el crecimiento de bacterias [27].

\section{POTENCIAL BACTERIANO INTRÍNSECO RELACIONADO CON LA CONTAMINACIÓN DE DESINFECTANTES DEL ÁREA QUIRÚRGICA}

El incremento en el uso de antibióticos y la respectiva presión selectiva que ejercen, es el factor más importante que hoy se reconoce como contribuyente a la aparición de diversas clases de resistencia bacteriana [28-29]. En este mismo sentido, actualmente se ha obtenido un avance considerable en la comprensión de la respuesta de las bacterias a los desinfectantes. La resistencia puede ser una propiedad natural de un organismo (intrínseca) o conseguida por mutación o adquisición de plásmidos (autorreplicación, ADN extracromosómico) o transposones (cromosomal o integrado en plásmidos, cassettes de ADN transmisibles). Los genes de resistencia naturales en plásmidos, se originan como mutaciones puntuales en los genes blanco (sitios de inserción de los genes de resistencia) de bacterias susceptibles y también de genes que les proveen protección contra otras bacterias [30-31].

\subsection{RESISTENCIA INTRÍNSECA DE LAS BACTERIAS GRAM NEGATIVAS}

La resistencia intrínseca se ha demostrado para bacterias gramnegativas, microorganismos esporulados, micobacterias y bajo ciertas condiciones en especies del 
género Staphylococcus. Las bacterias gramnegativas por lo general son más resistentes a los desinfectantes que las grampositivas no esporuladas. Se han hecho estudios donde se midieron las concentraciones mínimas inhibitorias (CIM) que presentan tanto las grampositivas como las gramnegativas, y se estableció que hay diferencias marcadas entre $S$. aureus y $E$. coli. Algunos bacilos gramnegativos no fermentadores como Pseudomonas aeruginosa y otros como Proteus $s p$ son los más resistentes a la mayoría de estos agentes incluida la Clorhexidina [32].

La membrana externa de las bacterias gramnegativas como $P$. aeruginosa actúa como una barrera que limita la entrada de varios tipos de agentes antibacterianos y es la responsable de su resistencia. Las moléculas hidrofílicas de bajo peso molecular pasan fácilmente a través de las porinas, en cambio las moléculas hidrofóbicas se difunden a través de la bicapa de la membrana [33]. $P$. aeruginosa presenta diferencias en la composición del lipopolisacarido (LPS) y el contenido de cationes como el magnesio, que produce enlaces estables entre moléculas de LPS y como complemento a este mecanismo, esta bacteria presenta porinas pequeñas que impiden el paso por difusión de ciertas sustancias desinfectantes [34]. En contraste Pseudomona stutzeri es muy sensible a muchos biocidas lo que implica que tales agentes tienen poca dificultad en atravesar su membrana externa. Estos datos permiten conocer que no son los componentes de la membrana externa los asociados a la resistencia de estas bacterias, sino los cambios en la disposición estructural de esta envoltura lipídica y que la membrana interna no juega ningún papel en dicha resistencia [35].

\subsection{RESISTENCIA INTRÍNSECA DE LA BACTERIAS GRAM POSITIVAS}

La pared celular de algunos cocos gram positivos, está compuesta de peptidoglucano y ácidos teicóicos, sin embargo, a pesar del grosor de la pared esta no representa una barrera efectiva contra los biocidas, lo que explica la sensibilidad de estos microorganismos a antisépticos como la clorhexidina y los compuestos de amonio cuaternario. En la naturaleza $S$. aureus puede existir en forma de cepas mucoides, rodeadas de una capa denominada slime. En los hospitales estas cepas están asociadas a infecciones relacionadas con el cuidado de pacientes (colonización de catéteres, flebitis y bacteriemia). El slime protege a las bacterias no solo de la acción de los anticuerpos y leucocitos sino también de algunos antibióticos y biocidas; en este caso actúa como una barrera física a la penetración de estos, ya sea interaccionando o absorbiéndolos [36]. No existe evidencia de que las bacterias gram positivas resistentes a antibióticos, sean resistentes a desinfectantes o antisépticos, sin embargo, algunos Enterococos son más resistentes a la acción de biocidas que los Estafilococos.

\subsection{RESISTENCIA INTRÍNSECA DE LAS MICOBACTERIAS}

La pared de las micobacterias es una estructura hidrofóbica con un esqueleto de micoarabinogalactano-peptidoglucano El peptidoglucano está unido covalentemente a un polisacárido (arabinogalactano) formado por arabinosa y galactosa esterificados a ácidos micólicos. La razón para esta resistencia es la impermeabilidad de la pared, principalmente por el alto contenido lipídico y la presencia de arabinogalactano, que limitan la penetración de los biocidas en el interior de la célula. No existe evidencia de que se produzca degradación enzimática de biocidas, ni se ha demostrado la existencia 
https://doi.org/10.22519/21455333.302

de resistencia mediada por plásmidos y transposones. Las micobacterias no tuberculosas están más expuestas a sustancias antibióticas naturales que las micobacterias patógenas primarias como $M$. tuberculosis, siendo más resistentes intrínsecamente a las moléculas biocidas aunque los mecanismos involucrados sean aún desconocidos [37]. Recientemente, se han descrito cepas de $M$. chelonae aisladas de endoscopios con alta resistencia a glutaraldehido [38]. Esta resistencia se explicaría por un descenso de los monosacáridos que forman los arabinomananos de la pared celular y que reducen la permeabilidad de dicha pared y por tanto la captación de glutaraldehido [39].

\subsection{RESISTENCIA INTRÍNSECA DE BACTERIAS ESPORULADAS}

Las cubiertas y el córtex de la espora tienen un papel fundamental en la resistencia como barreras de permeabilidad, y están implicadas, aunque parcialmente, en la resistencia a algunos antisépticos [40]. La resistencia al glutaraldehido está ligada a la formación de cubiertas. Otros dos aspectos importantes en la repercusión en la resistencia intrínseca de las esporas a los biocidas, son por un lado la supervivencia o el revival de las esporas afectadas por los biocidas, y por otro, el efecto de dichos biocidas sobre las esporas germinantes. El revival de las esporas tratadas con desinfectantes ha sido poco estudiado, aunque se conoce que varios agentes inhiben la germinación entre ellos los alcoholes, aldehídos, fenoles y cresoles. Los efectos de estos inhibidores pueden ser reversibles debido a que la unión de estas sustancias a la superficie de la espora es muy débil, ya que un lavado es suficiente para que no se produzca la acción del inhibidor.

\section{RESISTENCIA BACTERIANA ADQUIRIDA RELACIONADA CON LA CONTAMINACIÓN DE DESINFECTANTES DEL ÁREA QUIRÚRGICA}

Como se ha visto en los antibióticos y en los agentes quimioterapéuticos, la resistencia adquirida a los antisépticos y desinfectantes surge por mutación o por la adquisición de material genético en forma de plásmidos o transposones; estas configuraciones permiten grandes arreglos de genes de resistencia para la mayoría de los antibióticos y desinfectantes al ser transferidos juntos en un solo evento de conjugación [30]. La evaluación del papel de los plásmidos en la resistencia codificada (o incremento en la tolerancia) a los desinfectantes concluyó que los plásmidos no eran los responsables por los altos niveles de resistencia a desinfectantes de ciertas especies o cepas. Sin embargo, algunos autores evidencian la relación entre la presencia de plásmidos en bacterias con el aumento de la tolerancia a clorhexidina, CAC, triclosán, así como a diamidinas [41].

Se han visto altos niveles de resistencia en aislados de hospitales, aunque no es claro que haya una resistencia mediada por el plásmido [42]. También se ha documentado la participación de las bombas de eflujo en la adquisición de esta resistencia. La activación de estas bombas es mediada por plásmidos y es un importante mecanismo de resistencia a antibióticos, metales, desinfectantes y antisépticos catiónicos [43-44]. Los aislados de bacterias gramnegativas de hospitales son menos sensibles a los desinfectantes que las cepas de laboratorio. Debido a que las transferencias mediadas por plásmidos se han descartado aparentemente, la selección y la mutación podrían jugar un papel muy importante en la presencia de estos aislados. Las concentraciones 
https://doi.org/10.22519/21455333.302

subinhibitorias de antibióticos pueden causar cambios sutiles en la estructura externa de la bacteria, y estimular de esta forma el contacto célula a célula; queda el 
interrogante si las concentraciones residuales de antisépticos y desinfectantes en ambientes clínicos podrían producir el mismo efecto [45].

\section{CONCLUSIÓN}

La utilización de desinfectantes en el área quirúrgica, involucra una serie de protocolos y estrategias creadas con el objetivo de disminuir la adquisición y diseminación de infecciones del sitio quirúrgico. Se ha documentado la eficacia de estos compuestos en los procesos de desinfección por inmersión de material diagnóstico e instrumental quirúrgico usado en cirugía. Sin embargo, la presencia de células bacterianas viables en estas soluciones, hace pensar ¿Cuál es la real fuente de contaminación? ya que se pone en riesgo la vida del paciente por la consecuente introducción de gérmenes patógenos que pueden conducir a infección. Identificar los principales factores extrínsecos, intrínsecos y adquiridos, es el primer paso para definir propuestas claras de prevención y desarrollo de procedimientos quirúrgicos seguros.

En la identificación de factores extrínsecos es importante la implementación de estudios en los que se trabaje con muestras de desinfectantes tomadas de instituciones hospitalarias de diferente nivel de atención medica y en las que los protocolos de manejo de estas sustancias, evidencien diferencias significativas, que ayudan a definir el posible factor extrínseco relacionado. Es importante verificar que las cepas aisladas no presentan patrones de resistencia a antibióticos; porque esto involucraría factores intrínsecos o patrones adquiridos bacterianos, todo esto considerando que actualmente los mecanismos que genéticamente se activan contra los antibióticos, también se activan contra los desinfectantes por un mecanismo de resistencia cruzada. Al final atribuir a un solo grupo de factores la contaminación bacteriana de desinfectantes usados en el área quirúrgica, es inconveniente por lo que se requiere prestar una especial atención a cada uno de ellos dependiendo del contexto y las condiciones de cada institución de salud.

\section{BIBLIOGRAFÍA}

1. Rutala WA, Weber DJ, and Healthcare Infection Control Practices Advisory Committee. Guideline for Disinfection and Sterilization in Healthcare Facilities, CDC, 2008. Disponible en: http://www.cdc.gov/hicpac/pdf/guidelines/Disinfection Nov 2008.pdf

2. Álvarez C, Cortés J, Gómez C, Fernández J, Sossa M, Beltrán F, et al. Guías de práctica clínica para la prevención de infecciones intrahospitalarias asociadas al uso de dispositivos médicos. Infectio. 2010; 14:292-308.

3. Ortiz J, Athié J, Martínez V. Contaminación bacteriana potencialmente patógena en el manejo de la vía aérea en el Hospital Ángeles Mocel. Acta Médica Grupo Ángeles 2011; 9: 63-68.

4. Weber DJ, Rutala WA. Use of germicides in the home and the healthcare setting: Is there a relationship between germicide use and antibiotic resistance? Infect Control Hosp Epidemiol 2006; 27:1107-1119.

5. Prescott LM, Harley JP, Klein DA. Microbiología. 5a ed. Madrid: McGraw Hill Interamericana, 2004.http://books.google.es/books?id=MfW3TuHLa4gC\&pg=PA223\&lpg=PA223\&dq=Pres cott+LM,+Harley+JP,+Klein+DA.+Microbiolog\%C3\%ADa.+5a+ed.+Madrid:McGraw+Hill +Interamericana;+2004.\&source=bl\&ots=4n_gRIUUsG\&sig=sk3Psi_4BK5CnTuJsve3OFkL GY\&hl=es\&ei=AESKTqSvPIS2twed17WWA 
6. Rutala WA, Weber DJ. New Disinfection and Sterilization Methods. Emerging Infectious Diseases 2001; 7: 348-353.

7. Nachón F, Díaz J, Rivas V, González J, Nachón MG, Gracía F, et al.. Esterilización por inmersión. Estudio comparativo entre glutaraldehido al $2 \%$, agua electrolizada superoxidada con pH neutro y solución electrolizada por selectividad iónica con pH neutro. Rev Med UV, 2008; 8: 5-10.

8. Culver D, Gordon S, Mehta A. Infection control in the Bronchoscopy Suite. A review of outbreaks and CSV: Vol. 3 No.1 Año 2011

guidelines for prevention. Am J Respir Crit Care Med 2003; 167:1050-1056.

9. Repáraz F, Arina P, Artajo P, Sánchez MT, Escobar E. Limpieza y desinfección en el hospital. ANALES Sis San Navarra 2000, 23(Supl. 2).

10. Ayliffe G; minimal Access Therapy Decontamination Working Group. Decontamination of minimally invasive surgical endoscopes and accessories. J Hosp Infect 2000; 45: 263-267. Disponible en: http://www.ncbi.nlm.nih.gov/pubmed?term=Ayife\%20G.\%20et\%20al.\%20Decontamina\%F4\%80\%86 \%9Fon\%20of\%20minimally\%20invasive\%20surgical\%20and\%20accessories.\%20J\%20Hosp\%20Infe ct\%202000\%3B\%2045\%3A\%20263-277.

11. Hidalgo R, Zambrano A, Mireles $C$, Chiroles S, Villavicencio O. Aplicación de nuevo producto esterilizante (ADASPOR) en servicios de Microcirugía Oftalmológica. Rev Cubana Invest Bioméd 2011;30: 226-234.

12. Rutala WA, Weber DJ. Surface disinfection: should we do it? J Hosp Infect 2001;48: S64-S68. Disponible en: http://www.sciencedirect.com/science/article/pii/S0195670101900179

13. Maya J, Jamil S, Pacheco R, Valderrama S, Villegas M. Papel de la clorhexidina en la prevención de las infecciones asociadas a la atención en salud. Infectio. 2011;15: 98-107.

14. Weber DJ, Rutala WA, Sickbert-Bennett EE. Outbreaks associated with contaminated antiseptics and disinfectants. Antimicrob.Agents Chemother, 2007; 51: 4217-4224.

15. Herruzo-Cabrera R, Uriarte MC, Rey-Calero J. Antimicrobial effectiveness of $2 \%$ glutaraldehyde versus other disinfectants for hospital equipment, in an in vitro test based on germ-carriers with a high microbial contamination. Rev Stomatol Chir Maxillofac 1999;100: 299-305. Disponible en: http://pubget.com/paper/10672649?cb=1317303534\&cb=1325351053

16. Villagrana J, Solano R, Ibarra V, Sosa I, García C, Ahued J. Análisis microbiológico de la reutilización de trócares de laparoscopia en un hospital de tercer nivel. Perinatol Reprod Hum 2002; 16:96-102.

17. Leelaporn A, Paulsen I, Tennent J, Littlejhon T, Skurray R. Multidrug resistance to antiseptics and disinfectants in coagulase-negative staphylococci. J. Med. Microbiol. 1994; 40: 214-220.

18. Gajadhar T, Lara A, Sealy P, Adesiyun A. Microbial contamination of disinfectants and antiseptics in four major hospitals in Trinidad. Rev. Panam Salud Publica. 2003; 14: 193-200.

19. Raufman JP, Straus EW. Endoscopy procedures in the AIDS patient: risk, precautions, indications and obligation. Gastroenterol Clin North Am 1988; 17: 495-506. Disponible en: http://www.ncbi.nIm.nih.gov/pubmed?term=aufman\%2C\%20J.\%20P.\%20\%26\%20Straus\%2C\%20E. \%20\%E2\%80\%9CEndoscopy\%20Procedures\%20in\%20the\%20AIDS\%20patient.\%20Risk\%2C\%20p recautions \%2C\%20indications\%20and\%20obligation\%E2\%80\%9D\%2C\%20en\%20Gastroenterology $\% 20$ Clinics\%20of

20. Bonilla A, Pérez J. Aislamiento y caracterización fenotípica de microorganismos presentes en la sala de partos de un hospital de primer nivel del departamento de Cundinamarca. Pontificia Universidad Javeriana. Facultad de Ciencias. Bacteriología. Bogotá D. C., 2008. Disponible en: http://www.javeriana.edu.co/biblos/tesis/ciencias/tesis193.pdf

21. Secretaria Distrital de Salud de Bogotá. Guías para la prevención control y vigilancia epidemiológica de infecciones intrahospitalarias. 7 Uso de desinfectantes. Esfera Editores Ltda. 2004. Disponible en: http://www.saludcapital.gov.co/sitios/VigilanciaSaludPublica/Todo\%20IIH/007\%20Desinfectantes.pdf

22. Rodríguez A. La desinfección-antisepsia y esterilización en instituciones de salud. Atención primaria. Rev Cubana Med Gen Integr 2006; 22: 1-11.

23. Danchaivijitr S, Dhiraputra C, Rongrungruang Y, Srihapol N, Pumsuwan V. Microbial contamination of antiseptics and disinfectants. J Med Assoc Thai, 2005; 88: S133-S138.

24. Olayemi AB, Obayan Y. Contaminated disinfectants in health clinics in Ilorin, Nigeria. Infect Control Hosp Epidemiol 1994; 15: 581-582. Disponible en: http://www.ncbi.nlm.nih.gov/pubmed/7989730

25. Oie S, Kamiya A. Microbial contamination of antiseptics and disinfectants. Am J Infect Control 1996; 24: 389-395. 
http://www.ncbi.nlm.nih.gov/pubmed?term=Oie\%20S\%2C\%20Kamiya\%20A.\%20Microbial\%20conta mination\%20of\%20antiseptics\%20and\%20disinfectants.\%20Am\%20J\%20Infect\%20Control\%201996 \%3B24\%3A389\%E2\%80\%93395.

26. Padovani CM, Graziano KU, Goveia V. Evaluación microbiológica de las diferentes formulaciones antisépticas, polivinil-pirrolidona-yodo y clorhexidina, después de la contaminación intencional de los recipientes. Rev Latino-am Enfermagem 2008; 16: 106-110.

27. Jones BL, Gorman LJ, Simpson J, Curran ET, McNamee S, Lucas C, et al. An outbreak of Serratia marcescens in two neonatal intensive care units. J Hosp Infect 2000; 46: 314-319. Disponible en: Lordhttp://www.ncbi.nlm.niuy $\mathrm{G}$, Jaime h.gov/pubmed?term=Jones\%20BL\%2C\%20Gorman\%20LJ\%2C\%20Simpson\% 20J\%2C\%20Curran\%20ET\%2C\%20McNamee\%20S\%2C\%20Lucas\%20C.\%20An\%20outbreak\%20 of\%25qq20Serratia\%20marcescens\%20in\%20two\%20neonatal\%20intensive\%20care\%20units.\%20 J $\% 20 \mathrm{Hosp} \% 20$ Infect $\% 202000$

28. Ang JY, Ezike E, Asmar BI. Antibacterial resistance. Indian J Pediatr 2004; 71: 229-239. Disponible en: http://www.ncbi.nlm.nih.gov/pubmed?term=Ang\%20JY\%2C\%20Ezike\%20E\%2C\%20Asmar\%20BI.\% 20Antibacterial\%20resistance.\%20Indian\%20J\%20Pediat\%202004\%3B\%2071\%3A\%20229-239.

29. Levy SB Marsahll B. Antibacterial resistance worldwide: Causes, challengesand responses. Nat Med 2004; 10: S122-S129. Disponible en: http://www.ncbi.nlm.nih.gov/pubmed?term=Levy\%20SB.\%20Antibacterial\%20resistance\%20worldwid e\%3A\%20Causes\%2C\%20challengesand\%20responses.\%20Nature\%20Med\%202004\%3B\%2010\% 20(Suppl)\%3A\%20122-129.

30. Frost LS, Leplae R, Summers AO, Toussanint A. Mobile genetic elements: The agents of open source evolution. Nat Rev Microbiol 2005; 3: 722-732.Disponible en: http://www.ncbi.nlm.nih.gov/pubmed/16138100

31. McDonnell J, Russell D. Antiseptics and Disinfectants: Activity, Action, and Resistance. Clin Microbio Rev 1999; 12: 147-179. Disponible en: http://cmr.asm.org/content/12/1/147.full

32. Gilbert $P$, McBain AJ. Potential impact of increased use of biocides in consumer products on prevalence of antibiotic resistance. Clin Microbiol Rev 2003; 16: 189-208.

33. Sheldon A. Antiseptic "resistance": real or perceived threat? Clin Infect Dis 2005; 40: 1650-1656.

34. Cox AD, Wilkinson SG. Ionizing groups in lipopolysaccharides of Pseudomonas cepacia in relation to antibiotic resistance. Mol Microbiol 1991; 5: 641-646. Disponible en: http://www.ncbi.nlm.nih.gov/pubmed?term=Cox\%20AD\%2C\%20Wilkinson\%20SG.\%20lonizing\%20gr oups\%20in\%20lipopolysaccharides\%200f\%20Pseudomonas\%20cepacia\%20in\%20relation\%20to\%2 0antibiotic\%20resistance.\%20MolMicrobiol\%201991\%3B\%205\%3A\%20641-646.

35. Hernández-Rodriguez A. Aportaciones al estudio de la actividad antimicrobiana de los antisépticos y desinfectantes. Universidad Autónoma de Barcelona. Departamento de Genética y Microbiología. 2006. Disponible en: http://tdx.cat/bitstream/handle/10803/3898/ahr1de1.pdf?sequence=1

36. Seaman P, Day M, Denver A, Dietmar O. Susceptibility of capsular Staphylococcus aureus strains to some antibiotics, triclosan and cationic biocides. J Antimicrob Chemother Advance Access publication 4 August 2004.Disponible en: http://jac.oxfordjournals.org/content/54/3/696.full.pdf+html

37. Frenzel E, Schmidt S, Niederweis M, Steinhaue K. Importance of porins for biocide efficacy against Mycobacterium smegmatis. Appl. Environ. Microbiol 2011; 77: 3068-3073.

38. Manzoor SE, Lambert PA, Griffiths PA, Gill MJ, Fraise AP. Reduced glutaraldehyde susceptibility in Mycobacterium chelonae associated with altered cell wall polysaccharides. J Antimicrob Chemother. 1999; 43: 759-765.

39. Fraud S. Maillard JY, Russell AD. Comparison of the mycobactericidal activity of ortho- phthalaldehyde, glutaraldehyde and other dialdehydes by a quantitative suspension test. J Hosp Infect. 2001; 48: 214221. Disponible en: http://www.ncbi.nlm.nih.gov/pubmed/11439009

40. Mehmi M, Marshall LJ, Lambert PA, Smith JC. Evaluation of disinfecting procedures for aseptic transfer in hospital pharmacy departments. PDA J Pharm Sci Technol. 2009; 63: 123-138. Disponible en: http://www.ncbi.nlm.nih.gov/pubmed/19634351

41. Reynaldo MB, Flores MB, Viegas J, Magariños MC. Eficacia de algunos biocidas contra estafilococos hospitalarios sensibles y resistentes a la meticilina en la provincia de Buenos Aires, Argentina. $\underline{\text { Rev }}$ Panam Salud Publica. 2004; 16: 187-192. 
https://doi.org/10.22519/21455333.302

42. Rosenblatt-Farrell, N. El paisaje de la resistencia a los antibióticos. Salud pública Méx 2009; 51: 435442.

43. Russell AD. Antibiotic and resistance in bacteria: Introduction. J Appl Microbiol 2002; 92:1S-3S.

44. Russell AD. Bacterial adaptation and resistance to antiseptics, disinfectants and preservatives is not a new phenomenon. Journal of Hospital Infection 2004; 57: 97-104.

45. Cabrera CE; Gómez RF, Zúñiga AE. La resistencia de bacterias a antibióticos, antisépticos y desinfectantes una manifestación de los mecanismos de supervivencia y adaptación. Colomb. Med. 2007; 38: 149-158.

CSV: Vol. 3 No.1 Año 2011. 